\title{
Cancer upregulated gene 2, a novel oncogene, enhances migration and drug resistance of colon cancer cells via STAT1 activation
}

\author{
WARAPORN MALILAS ${ }^{1}$, SANG SEOK KOH ${ }^{2}$, SEOKHO KIM ${ }^{2}$, RATAKORN SRISUTTEE ${ }^{1}$, IL-RAE CHO $^{1}$, \\ JEONG MOON $^{1}$, HWA-SEUNG YOO ${ }^{3}$, SANGTAEK OH ${ }^{4}$, RANDAL N. JOHNSTON ${ }^{5}$ and YOUNG-HWA CHUNG ${ }^{1}$ \\ ${ }^{1}$ WCU, Department of Cogno-Mechatronics Engineering, Pusan National University, Busan 609-735; ${ }^{2}$ Immunotherapy \\ Research Center, Korea Research Institute of Bioscience and Biotechnology, and Department of Functional Genomics, \\ University of Science and Technology, Daejeon 305-333; ${ }^{3}$ East-West Cancer Center, College of Oriental Medicine, \\ Daejeon University, Daejeon 300-716; ${ }^{4}$ Department of Advanced Fermentation Fusion Science and Technology, \\ Kookmin University, Seoul 136-702, Republic of Korea; ${ }^{5}$ Department of Biochemistry and \\ Molecular Biology, University of Calgary, Calgary T2N4N1, Alberta, Canada
}

Received April 1,2013; Accepted May 28, 2013

DOI: 10.3892/ijo.2013.2049

\begin{abstract}
Cancer upregulated gene (CUG) 2, as a novel oncogene, has been predominantly detected in various cancer tissues, such as ovary, liver, lung and colon. We recently showed that CUG2 elevates STAT1 activity, leading to resistance to infection by oncolytic vesicular stomatitis virus. To investigate a possible role for CUG2-induced activation of STAT1 in oncogenesis, we first established a colon cancer cell line stably expressing CUG2 (Colon26L5-CUG2). Colon26L5-CUG2 exhibited higher levels not only in phosphorylation of STAT1, but also phosphorylation of Jak1/Tyk2 compared to that of the control (Colon26L5-Vec) cell line. Inhibition of Akt or ERK activity reduced phosphorylation of STAT1 in Colon26L5-CUG2 cells whereas inhibition of p38 MAPK did not significantly decrease levels of STAT1 phosphorylation, indicating that cell proliferation signals may be involved in CUG2-mediated activation of STAT1. Suppression of STAT1 expression diminished cell migration and wound healing compared to the control cells. In addition, since CUG2 expression conferred resistance to DNA damage caused by doxorubicin treatment, we investigated whether STAT1 is involved in resistance to doxorubicininduced cell death. We found that STAT1 was not activated in Colon26L5-Vec cells while phosphorylated STAT1 was maintained in Colon26L5-CUG2 cells during doxorubicin treatment. Furthermore, suppression of STAT1 expression
\end{abstract}

Correspondence to: Professor Young-Hwa Chung, WCU, Department of Cogno-Mechatronics Engineering, Pusan National University, 30 Jangjeon-dong, Geumjeong-gu, Busan 609-735, Republic of Korea

E-mail: younghc@pusan.ac.kr

Key words: cancer upregulated gene 2, STAT1, cell migration, drug resistance sensitized Colon26L5-CUG2 cells to doxorubicin-induced apoptosis whereas the control cells exhibited resistance to doxorubicin. Taken together, our results suggest that CUG2 enhances metastasis and drug resistance through STAT1 activation, which eventually contributes to tumor progression.

\section{Introduction}

To discover new genes that play a crucial role in common tumorigenesis regardless of tissue origin, we analyzed commonly upregulated unknown genes in 242 normal and 300 tumor samples originating from 11 different tissues using the Affymetrix gene chip system. An Affymetrix fragment, later named cancer upregulated gene (CUG) 2 was identified as a candidate gene that is commonly upregulated in various tumor tissues such as ovary, liver, lung and colon. This CUG2 was mapped to chromosome $6 \mathrm{q} 22.32$; it spans $\sim 8.5 \mathrm{~kb}$ with a three-exon structure and encodes a 88 -amino acid polypeptide (1). Further study has revealed that CUG2 is a new component of centromere required for a proper kinetochore function during cell division (2). Of interest, CUG2 has been shown to harbor an oncogenic effect in a transplanted model using NIH3T3 cells expressing CUG2, in a manner similar to Ras (1). Although CUG2 overexpression leads to activated Ras and MAPKs including p38 MAPK, which eventually facilitates oncolytic reoviral replication (3), CUG2 contrastingly provides resistance to oncolytic vesicular stomatitis virus infection through activation of STAT1 as shown in our recent study (4).

Although STAT1 is well known as a master transcription factor for IFN-related intracellular signaling, leading to antiviral activity, several lines of evidence have shown that STAT1 plays a role as an anti-oncogenic molecule in part by upregulation of caspases $(5,6)$, cyclin-dependent kinase inhibitor 1A (7), the IFN-regulatory Factor 1 (IRF1)/p53 pathway (8), and downregulation of the BCL2 family (9). In contrast, emerging data reveal that in certain cellular contexts the IFN/STAT1 pathway may facilitate tumor cell growth. 
Other studies have reported that resistance to ionizing radiation and IFNs is associated with constitutive overexpression of the IFN/STAT1 pathway in radio-resistant tumor cells (10). Recent studies have also demonstrated that constitutive overexpression of STAT1 is positively correlated with protection of tumor cells from genotoxic stress such as treatment with doxorubicin (11), or cisplatin (12). Furthermore, since overexpression of the IFN/STAT1 pathway is associated with poor prognosis in different types of cancer, the IFN-related genes are suggested as predictive markers for breast cancer patients resistant to the adjuvant chemotherapy (13).

This study was initiated to explore further the possible biological consequence of the activation of STAT1 mediated by CUG2 during the development of cancer. We herein report that the CUG2-induced activation of STAT1 promotes both enhanced cell migration and resistance to an anticancer drug such as doxorubicin, eventually contributing to the metastasis and progression of cancer.

\section{Materials and methods}

Cell cultures. Colon26L5 cells, derived from mouse colon cancer and modified so that they stably express either vector alone (Colon26L5-Vec) or CUG2 (Colon26L5-CUG2), were cultured in DMEM supplemented with 10\% FBS, $1 \%$ penicillin and $1 \%$ streptomycin, puromycin (Sigma-Aldrich, St. Louis, $\mathrm{MO} ; 2 \mu \mathrm{g} / \mathrm{ml}$ ) under $37^{\circ} \mathrm{C}$ and $5 \% \mathrm{CO}_{2}$. In addition, Colon26L5-CUG2 cells stably suppressing STAT1 using shRNA (Colon26L5-CUG2-shSTAT1) and the control cells (Colon26L5-CUG2-shCon) were cultured in the same condition except with zeocin (Calbiochem, San Jose, CA, USA; $100 \mu \mathrm{g} / \mathrm{ml})$.

Reagents and antibodies. For inhibition of protein kinases, wortmannin, PD98059, SB203580 and Jak inhibitor I were purchased from Calbiochem. For immunoblotting, anti-STAT1, STAT2, Jak1, Tyk2 and their phospho-specific antibodies were acquired from Cell Signaling Biotechnology (Danvers, MA, USA). In addition, anti-caspase- 8 antibody was purchased from Cell Signaling Biotechnology. Anti- $\beta$-actin antibody was obtained from Santa Cruz Biotechnology (Santa Cruz, CA, USA) and anti-VSV glycoprotein (G) antibody was obtained from Abcam (Cambridge, MA, USA).

Western blotting. Cells were harvested and lysed with lysis buffer containing 1\% NP-40 and protease inhibitors (SigmaAldrich). For immunoblotting, proteins from whole cell lysates were resolved by 10 or $12 \%$ SDS-polyacrylamide gel electrophoresis (PAGE) and then transferred to nitrocellulose membranes. Primary antibodies were used at 1:1000 or 1:2000 dilutions, and secondary antibodies conjugated with horseradish peroxidase were used at 1:2000 dilutions in 5\% nonfat dry milk. After the final washing, the membranes were exposed for an enhanced chemiluminescence assay using the LAS 4000 mini (Fuji, Tokyo, Japan).

Short interference RNA transfection. Cells were trypsinized and incubated overnight to achieve $60-70 \%$ confluency before siRNA transfection. STAT1 siRNA (commercially pre-made at Bioneer, Daejeon, Korea) or a negative control siRNA
(Bioneer) were mixed with Lipofectamine 2000 (Invitrogen, Carlsbad, CA, USA). The cells were incubated with the transfection mixture for $6 \mathrm{~h}$ and then rinsed with DMEM containing $10 \%$ FBS. The cells were incubated for $48 \mathrm{~h}$ before harvest.

Cell migration assay. Migration assays were performed using 48-well Boyden chambers (Neuroprobe, Gaitherburg, MD, USA) as described elsewhere (14). The lower wells of the chamber were filled with standard culture media. The chamber was assembled using polycarbonate filters (Neuroprobe). Cells in serum-free media $\left(5 \times 10^{4}\right.$ cells/well) were seeded in the upper compartment of the chamber. After incubation for $24 \mathrm{~h}$, cell migration was quantified by counting the number of migrated cells after staining with hematoxylin and eosin.

Wound healing assay. Cell migration was assessed using a scratch wound assay (15). Briefly, Colon26L5-CUG2-shCon or Colon26L5-CUG2-shSTAT cells were cultured in sixwell plates $\left(5 \times 10^{5}\right.$ cells/well). When the cells were grown to $90 \%$ confluence, a single wound was made in the center of the cell monolayer using a $\mathrm{P}-200$ pipette tip. At 0 and $48 \mathrm{~h}$ of incubation, the wound closure areas were visualized by phase-contrast microscopy (Olympus, Tokyo, Japan) with a magnification of $\mathrm{x} 100$.

Reverse transcriptase-polymerase chain reaction (RT-PCR). Total RNA was extracted from cells using the RNeasy mini kit (Qiagen, Valencia, CA, USA) in accordance with the manufacturer's instructions. Total RNA $(3 \mu \mathrm{g})$ was converted to cDNA using Superscript II reverse transcriptase (Invitrogen), and PCR was performed using the specific primers (sense primer: 5'-GCGCTGTCGACCATAGTCTCCCAGAGG AAG-3'; anti-sense primer: 5'-CTAACCTCTGCTCTTCTT TAGAATTACCTTTGCTGC-3'). The cDNA products of each reaction were diluted, and PCR was run at the optimized cycle number. $\beta$-actin mRNA was used as an internal standard.

Statistical analysis. Data are presented as a means \pm standard deviation. Student's t-test was used for statistical analysis, with p-value $<0.05$ defined as significance.

\section{Results}

Akt-ERK signaling axis is involved in CUG2-mediated STAT1 activation. As we earlier reported that CUG2, a novel oncogene, induces activation of STAT1 in murine NIH3T3 fibroblast cells (4), we next decided to explore possible mechanisms by which the activation of STAT1 by CUG2 may be involved in the development of cancer. To address this question, we first established Colon26L5 cells, derived from a murine colon cell line, stably expressing a vector only (Colon26L5-Vec), or human CUG2 gene (Colon26L5-CUG2). Expression of human CUG2 transcripts were confirmed by RT-PCR in Colon26L5-CUG2 cells (Fig. 1A). We then observed that Colon26L5-CUG2 cells also exhibited higher levels of phosphorylated STAT1 that were higher than in controls, as we had seen earlier in NIH3T3 cells stably expressing CUG2 (NIHCUG2) cells. Furthermore, phosphorylation of Jak1 and Tyk2, downstream molecules of type I IFN signaling, was also seen when compared to Colon26L5-Vec cells (Fig. 1B). The results 


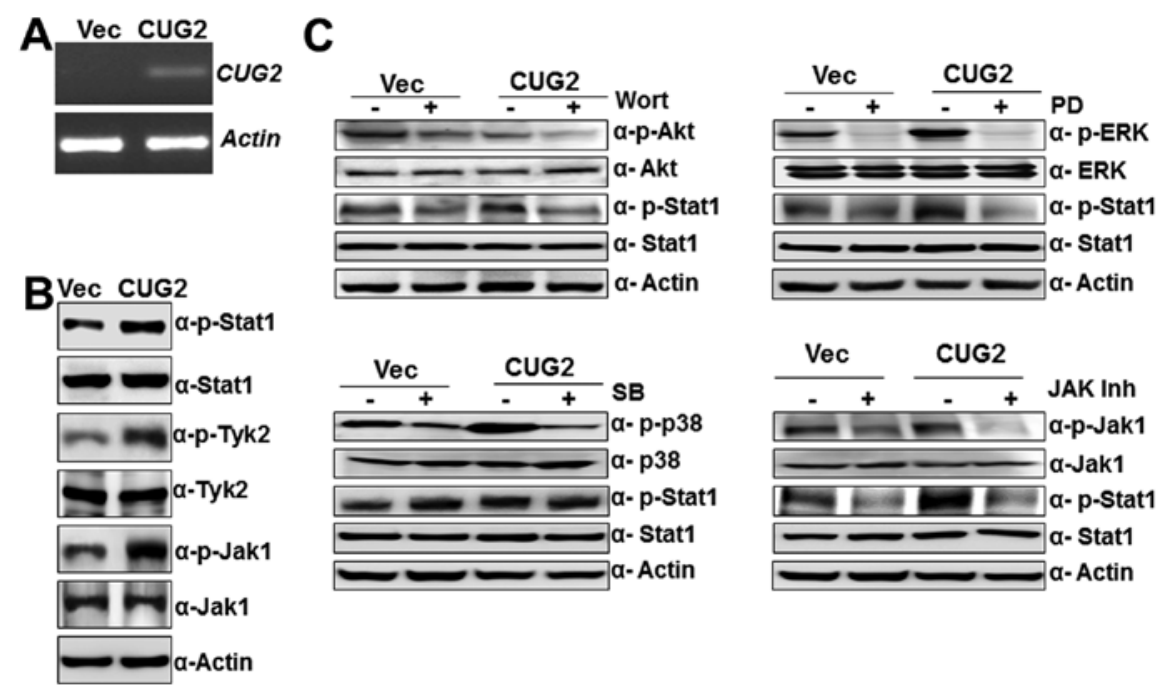

Figure 1. Akt-ERK signaling is involved in CUG2-mediated activation of STAT1 in Colon26L5 cells. (A) Total RNAs from Colon26L5-Vec and Colon26L5-CUG2 cells were isolated and human CUG2 transcript level was examined by RT-PCR. (B and C) Colon26L5-Vec and Colon26L5-CUG2 cells were untreated or treated with wortmannin (Wort; $7 \mu \mathrm{M}$ ), PD98059 (PD; $30 \mu \mathrm{M}$ ), SB203580 (SB; $30 \mu \mathrm{M}$ ) and Jak inhibitor I (JAK Inh; $80 \mu \mathrm{M}$ ) for $24 \mathrm{~h}$ and cell lysates separated by a $10 \%$ SDS-PAGE gel. STAT1, Akt, Jak1, Tyk2 and their phosphorylated proteins were detected by immunoblotting with the corresponding antibodies.
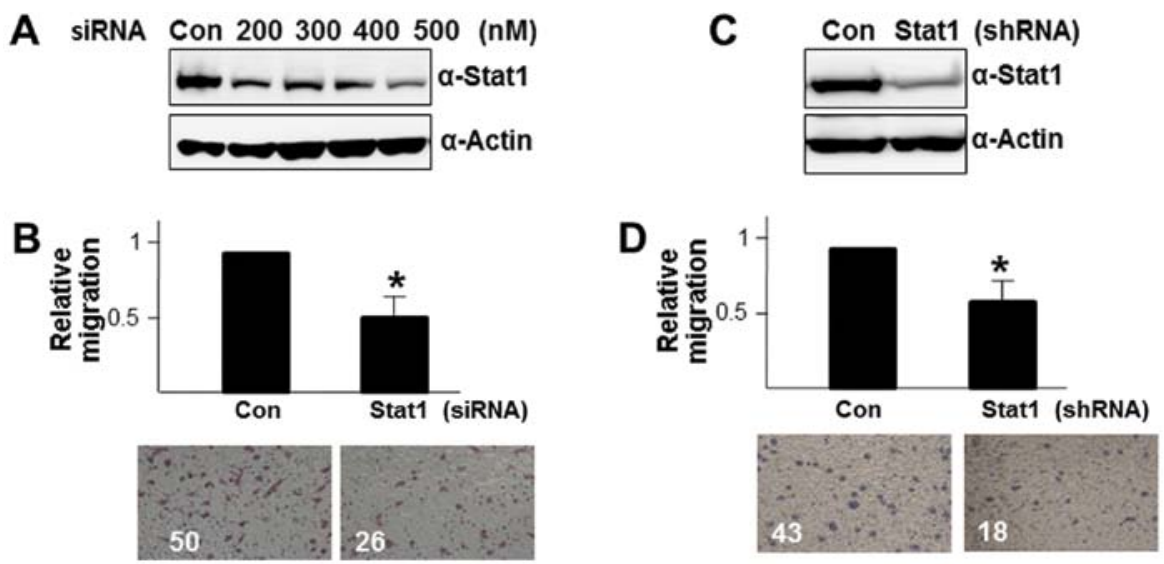

Figure 2. Suppression of STAT1 reduces migration of Colon26L5-CUG2 cells. (A and C) Colon26L5-CUG2 cells were treated with control or STAT1 siRNA for $48 \mathrm{~h}$ and the expression of STAT1 was examined by immunoblotting using its antibody. Suppression of STAT1 expression was examined in Colon26L5CUG2-shSTAT1 and Colon26L5-CUG2-ShCon cells by immunoblotting using an anti-STAT1 antibody. (B and D) At $24 \mathrm{~h}$ post-transfection with control or STAT1 siRNA in Colon26L5-CUG2 cells, the culture medium was replaced with serum-free medium. At $24 \mathrm{~h}$ post-subculture of Colon26L5-CUG2-shSTAT1 and Colon26L5-CUG2-shCon, the culture medium was replaced with serum-free medium. The cells in the serum-free well were placed on the serumcontaining well and incubated for $24 \mathrm{~h}$. Cell migration was quantified by counting the number of migrated cells after staining with hematoxylin and eosin. The assay was carried out in triplicate and error bars indicate standard deviation $\left({ }^{*} \mathrm{p}<0.05\right)$.

indicate that CUG2 expression activates STAT1 signaling in Colon26L5 cells without ligand stimulation.

We next explored which signaling pathways are involved in CUG2-mediated STAT1 activation. To answer this question, we treated Colon26L5-CUG2 cells with wortmannin (Akt inhibitor, $7 \mu \mathrm{M}$ ), $\mathrm{PD} 98059$ (ERK inhibitor, $30 \mu \mathrm{M}$ ), SB203580 (p38 MAPK inhibitor, $30 \mu \mathrm{M}$ ), or Jak1 inhibitor I (80 $\mu \mathrm{M})$. Inhibition of Jak1, an up-stream signaling molecule of STAT1, reduced phosphorylation of STAT1 in Colon26L5-CUG2 cells as expected (Fig. 1C). Moreover, we found that treatment with wortmannin and PD98059 significantly suppressed STAT1 phosphorylation whereas SB203580 treatment did not significantly reduce phosphorylation of STAT1 in Colon26L5-CUG2 cells (Fig. 1C), indicating that a cell proliferation signaling pathway is involved in CUG2-induced STAT1 activation, but stress-related signaling may not be.

CUG2-mediated STAT1 promotes migration and wound healing of Colon26L5 cells. To explore whether CUG2mediated activation of STAT1 is directly related to tumor progress and metastasis, we first examined cell migration in Colon26L5-CUG2 cells under suppression of STAT1. To address this issue, we transiently introduced STAT1 siRNA into Colon26L5-CUG2 cells and optimized the inhibitory concentration of STAT1 siRNA at $500 \mathrm{nM}$ (Fig. 2A). We then counted the cells migrated from serum-free medium to serumcontaining medium after transfection of Colon26L5-CUG2 cells with STAT1 siRNA. We observed that the number of 

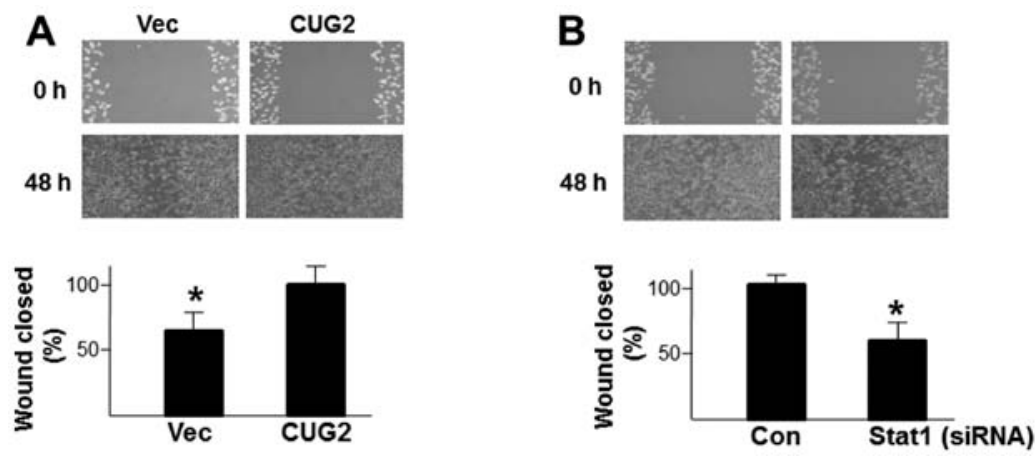

Figure 3. Suppression of STAT1 wound healing of Colon26L5-CUG2 cells. (A and B) Colon26L5-Vec, Colon26L5-CUG2 cells, and Colon26L5-CUG2 transfected with STAT1 or control siRNA cells were grown to confluence on 12-well tissue culture plates, and serum-starved for $24 \mathrm{~h}$. Cells were then gently scraped with a plastic tip to produce a clean $\sim 1$-mm-wide wound area and the wound closure areas were monitored by phase-contrast microscope with a magnification of x100. Cells were counted in the wound closure area using the Image-pro Plus program. The assay was carried out in triplicate and error bars indicate standard deviation $\left({ }^{*} \mathrm{p}<0.05\right)$.
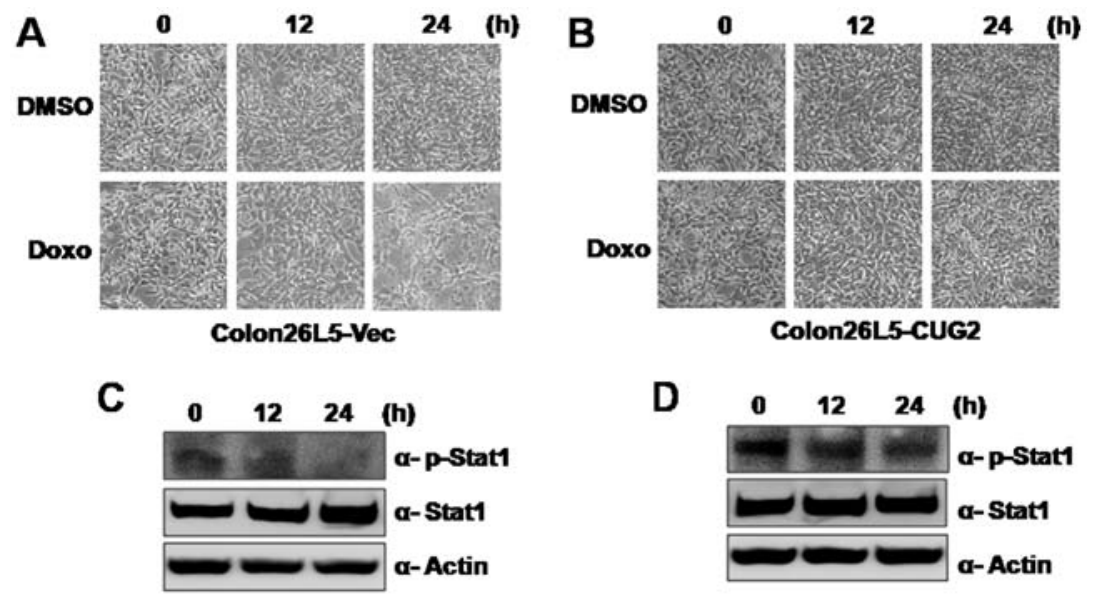

Figure 4. CUG2 confers resistance to doxorubicin-induced cell death. (A and B) Colon26L5-Vec and Colon26L5-CUG2 cells were treated with doxorubicin $(5 \mu \mathrm{M})$ for $24 \mathrm{~h}$. Cell viability was monitored under light microscopy. (C and D) Cell lysates from Colon26L5-Vec and Colon26L5-CUG2 cells treated with doxorubicin $(5 \mu \mathrm{M})$ were then prepared and phosphor-STAT1 was examined with immunoblotting using the corresponding antibody.

migrating Colon26L5-CUG2 cells transfected with STAT1 siRNA is significantly less than that of Colon26L5-CUG2 cells transfected with control siRNA in the serum-containing well ( $\mathrm{p}<0.05$; Fig. 2B), indicating that STAT1 expression is required for the efficient migration of colon cancer cells. To confirm this result, we introduced the shSTAT1 vector into Colon26L5-CUG2 cells and established Colon26L5-CUG2 cells stably suppressing STAT1 (Colon26L5-CUG2-shSTAT1; Fig. 2C). We thereafter compared cell migration between Colon26L5-CUG2-shSTAT1 and Colon26L5-CUG2-shCon cells as a control. We observed the same pattern of cell migration as seen in Colon26L5-CUG2 cells transiently transfected with STAT1 siRNA and control siRNA (p<0.05; Fig. 2D). In parallel experiments, we also observed that suppression of STAT1 expression with the shSTAT1 vector reduced migration of NIH-CUG2 cells (data not shown).

In addition, when we compared the capacity for cell locomotion during wound healing between Colon26L5-Vec and Colon26L5-CUG2 cells, we found that Colon26L5-CUG2 cells show faster healing than Colon26L5-Vec cells $(\mathrm{p}<0.05$; Fig. 3A). The result indicates that upregulation of CUG2 elevates the ability of wound healing in colon cells. To test whether STAT1 is a critical molecule in enhancing the ability of wound healing, we examined wound healing in Colon26L5 cells with suppression of STAT1 protein levels. We found that Colon26L5-CUG2 cells transfected with control siRNA exhibited faster healing from wounds than did Colon26L5-CUG2 cells transfected with STAT1 siRNA (p<0.05; Fig. 3B). Based on these results, we suggest that STAT1 is required for cell migration and wound healing of colon cancer cells overexpressing CUG2.

CUG2-mediated STAT1 activation confers Colon26L5 cells resistance to doxorubicin-induced apoptosis. To explore another possible biological consequence of STAT1 activation induced by CUG2, we examined whether upregulation of CUG2 provides resistance to an anticancer drug such as doxorubicin. When Colon26L5-Vec and Colon26L5-CUG2 cells were treated with doxorubicin, Colon26L5-Vec cells were highly susceptible to cell death during doxorubicin treatment (Fig. 4A). However, Colon26L5-CUG2 cells were resistant to doxorubicin (Fig. 4B). In addition, when we examined phosphorylation levels of STAT1 in Colon26L5-Vec and Colon26L5-CUG2 cells, we found that phosphorylation of STAT1 was decreased in Colon26L5-Vec cells while the phosphorylated STAT1 level was maintained in 

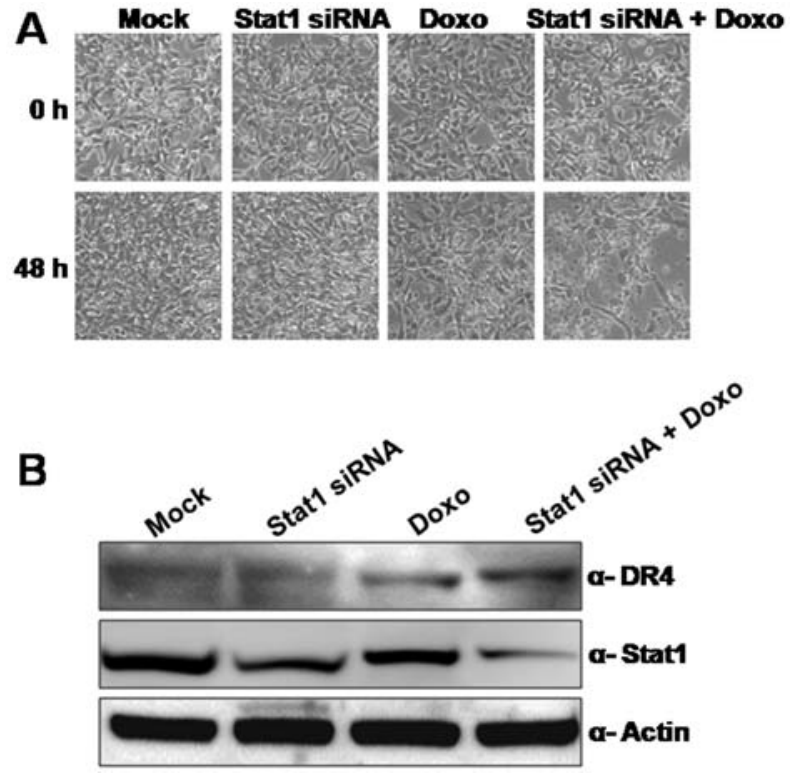

Figure 5. Suppression of STAT1 sensitizes doxorubicin-induced apoptosis in Colon26L5-CUG2 cells. (A) Colon26L5-CUG2 cells were treated with control siRNA or STAT1 siRNA $(500 \mathrm{nM})$ and then treated with doxorubicin $(5 \mu \mathrm{M})$ for $48 \mathrm{~h}$. Cell viability was monitored under light microscopy. (B) The lysates were then prepared and levels of STAT1 protein, and DR4 was evaluated by immunoblotting using the corresponding antibodies.

Colon26L5-CUG2 cells during doxorubicin treatment for $24 \mathrm{~h}$ (Fig. 4C and D). This result suggests that CUG2 confers resistance in Colon26L5 cells to doxorubicin through STAT1 activation.

To confirm whether CUG2-mediated activation of STAT1 affects survival of colon cancer cells under doxorubicin treatment, we treated Colon26L5-CUG2 cells with doxorubicin alone, STAT1 siRNA alone or doxorubicin plus STAT1 siRNA. Colon26L5-CUG2 cells survived in up to $500 \mu \mathrm{g} / \mathrm{ml}$ doxorubicin for $48 \mathrm{~h}$ (Fig. 5A). However, the combined treatment sensitized doxorubicin-induced cell death. We observed cell death beginning from $24 \mathrm{~h}$ post-treatment of doxorubicin under suppression of STAT1 expression (data not shown) and clearly found extensive cell death of Colon26L5-CUG2 cells at $48 \mathrm{~h}$. This cell death resulted from apoptosis induced by doxorubicin because increase of death receptor (DR) 4 expression was found in the co-treatment (Fig. 5B). Furthermore, we found that NIH-CUG2-shSTAT1 cells were more susceptible to doxorubicin than the NIH-CUG2-shCon cells (data not shown). These results indicate that STAT1 confers resistance to doxorubicin-induced apoptosis in Colon26L5-CUG2 cells. The result suggests that STAT may be an important anticancer therapeutic target in cancer cells overexpressing CUG2.

\section{Discussion}

Many studies have reported that STAT1 plays a role as tumor suppressor. For example, one study has shown that STAT1 is an important component of ErB2/Neu signaling that determines the transforming capacity of the receptor tyrosine kinase (16). STAT1 knockout mice were more susceptible to chemical carcinogenesis than their wild-type counterparts (17). Double knockout STAT1 ${ }^{-/}$, p53 ${ }^{-/-}$mice exhibited a higher incidence of tumor formation and a broader spectrum of tumors than p53 ${ }^{-/-}$knockout mice (17). Furthermore, STAT1 activation inhibited cell proliferation and enhanced the induction of apoptosis in tumor cells treated with IFN- $\gamma$ or TNF- $\alpha$ via upregulation of p21, a cyclin-dependent kinase inhibitor $(7,17)$. In Ras-transformed cells, activation of STAT1 also inhibited Ras-MAPK kinase signaling and reduced expression of RhoA, Cdc42, and Rac1 GTPases (18). In addition, STAT1 phosphorylation at tyrosine 701 activating $\mathrm{p} 27 \mathrm{Kip} 1$, a cyclin-dependent kinase inhibitor, has also been reported (19).

However, herein we add complexity to the earlier findings by reporting that $\mathrm{CUG} 2$ contributes to the tumor progression of colon cancer cells by the enhancement of cell migration and cell survival during DNA damage, apparently via activation of STAT1. Thus, we suggest that STAT1 can also play an unexpected role as a proto-oncogene, as we found that suppression of STAT1 with siRNA or shRNA vector reduced cell migration and enhanced doxorubicin-induced apoptosis in colon cells overexpressing CUG2. Our proposal is supported by other studies showing that constitutive activation of STAT1 accelerates metastasis of melanoma cancer cells and confers resistance to drug- or ionization-induced cell death of melanoma (20). Another doxorubicin-resistant myeloma cell line also exhibited a higher abundance of phosphorylated STAT1 (11).

STAT1 signaling was reported to be involved in resistance to the platinum drug AMD473 in ovarian cancer (12). Further studies showed that knockdown of STAT1 led to significant suppression of tumor growth and enhancement of sensitivity to irradiation and drug treatment compared to untreated tumor cells $(11,21)$. These changes were accompanied by alternative patterns of gene expression such as glycolysis/gluconeogenesis, the citric acid cycle, and oxidative phosphorylation. In particular, STAT1 expression had the most significant influence on the glycolysis/gluconeogenesis pathway, suggesting that STAT1-dependent expression of the energy metabolic pathway is associated with tumor growth and resistance to stress such as irradiation (22). We will therefore investigate metabolic pathways such as glycolysis/gluconeogeneis, the citric acid cycle, and oxidative phosphorylation in both Colon26L5-CUG2-shSTAT1 and Colon26L5-CUG2-shCon cells using microarrays in our next study.

We observed that CUG2 expression constitutively activates STAT1 in NIH3T3 and Colon25L5 cells and that the AktERK signaling pathway is involved in this event but p38MAPK is not. However, we do not know the detailed mechanism by which CUG2 stimulates STAT1 through Akt-ERK signaling. Of note, a recent study has shown that CUG2 interacts with nucleophosmin (NPM1/B23) protein (23). NPM1/B23 protein has multiple functions including cell growth, proliferation, resistance to stress and cancer development (24,25). Based on these findings, we speculate that CUG2, a component of kinetochores, plays a role in the development of cancer at least in part through its interaction with NPM1/B23 protein. Cancerous cells mediated by the CUG2-NPM1/B23 signaling axis produce growth factors and cytokines including EGF and IL-6, which can then deliver signals to the secretory or neighboring cells in an autocrine or a paracrine manner. Moreover, it is known that not only IFN signaling but also growth factor signaling (such as EGF) can activate STAT1 (26). Taken together, we propose that the interaction of CUG2 with 
NPM1/B23 protein can activate EGF release for cell proliferation, leading thereby to STAT1 activation. To answer more precisely how CUG2 overexpression might activate STAT1 through its interaction with NPM1/B23 protein will require further studies.

Since we demonstrated enhancement of cell migration as consequence of STAT1 activation by CUG2, we examined genes related to cell migration using microarray assays. We found that transcripts of CCL5, CCL9 and CXCL10 increased 12-, 6.8-, and 4.5-fold, respectively, in Colon26L5-CUG2 cells compared to those in Colon26L5-Vec cells (data not shown). Many lines of evidence have supported that CCL5, CCL9 and CXCL10 are involved in metastasis of various cancer cells (27-29), and have shown that IFN signaling induces upregulation of these chemokines (30-32). We therefore assume that upregulated chemokines through the STAT1-IFN axis enhance migration of colon cancer cells. Based on our results, we suggest that CUG2 enhances metastasis and drug resistance through STAT1 activation, which eventually contributes to tumor progression.

\section{Acknowledgements}

This study was supported by a grant from the National R\&D Program for Cancer Control, Ministry of Health and Welfare, Republic of Korea (1120140) and the World Class University Program (R31-2008-000-20004-0) through National Research Foundation funded by the Korean government.

\section{References}

1. Lee S, Gang J, Jeon SB, et al: Molecular cloning and functional analysis of a novel oncogene, cancer-upregulated gene 2 (CUG2). Biochem Biophys Res Commun 360: 633-639, 2007.

2. Kim H, Lee M, Lee S, et al: Cancer-upregulated gene 2 (CUG2), a new component of centromere complex, is required for kinetochore function. Mol Cells 27: 697-701, 2009.

3. Park EH, Park EH, Cho IR, et al: CUG2, a novel oncogene confers reoviral replication through Ras and p38 signaling pathway. Cancer Gene Ther 17: 307-314, 2010.

4. Malilas W, Koh SS, Srisuttee R, et al: Cancer upregulated gene 2, a novel oncogene, confers resistance to oncolytic vesicular stomatitis virus through STAT1-OASL2 signaling. Cancer Gene Ther 20: 125-132, 2013.

5. Chin YE, Kitagawa M, Kuida K, Flavell RA and Fu XY: Activation of the STAT signaling pathway can cause expression of caspase 1 and apoptosis. Mol Cell Biol 17: 5328-5337, 1997.

6. Kumar A, Commane M, Flickinger TW, Horvath CM and Stark GR: Defective TNF-alpha-induced apoptosis in STAT1null cells due to low constitutive levels of caspases. Science 278: 1630-1632, 1997

7. Chin YE, Kitagawa M, Su WC, You ZH, Iwamoto Y and Fu XY: Cell growth arrest and induction of cyclin-dependent kinase inhibitor p21 WAF1/CIP1 mediated by STAT1. Science 272: $719-722,1996$

8. Townsend PA, Scarabelli TM, Davidson SM, Knight RA, Latchman DS and Stephanou A: STAT-1 interacts with p53 to enhance DNA damage-induced apoptosis. J Biol Chem 279: 5811-5820, 2004.

9. Stephanou A, Brar BK, Knight RA and Latchman DS: Opposing actions of STAT- 1 and STAT- 3 on the Bcl-2 and Bcl-x promoters. Cell Death Differ 7: 329-330, 2000.

10. Khodarev NN, Beckett M, Labay E, Darga T, Roizman B and Weichselbaum RR: STAT1 is overexpressed in tumors selected for radioresistance and confers protection from radiation in transduced sensitive cells. Proc Natl Acad Sci USA 101: 1714-1719, 2004.
11. Fryknas M, Dhar S, Oberg F, et al: STAT1 signaling is associated with acquired crossresistance to doxorubicin and radiation in myeloma cell lines. Int J Cancer 120: 189-195, 2007.

12. Roberts D, Schick J, Conway S, et al: Identification of genes associated with platinum drug sensitivity and resistance in human ovarian cancer cells. Br J Cancer 92: 1149-1158, 2005.

13. Weichselbaum RR, Ishwaran H, Yoon T, et al: An interferonrelated gene signature for DNA damage resistance is a predictive marker for chemotherapy and radiation for breast cancer. Proc Natl Acad Sci USA 105: 18490-18495, 2008.

14. Park HD, Lee Y, Oh YK, et al: Pancreatic adenocarcinoma upregulated factor promotes metastasis by regulating TLR/CXCR4 activation. Oncogene 30: 201-211, 2011.

15. Liang CC, Park AY and Guan JL: In vitro scratch assay: a convenient and inexpensive method for analysis of cell migration in vitro. Nat Protoc 2: 329-333, 2007.

16. Raven JF, Williams V, Wang S, et al: Stat1 is a suppressor of ErbB2/Neu-mediated cellular transformation and mouse mammary gland tumor formation. Cell Cycle 10: 794-804, 2011.

17. Kaplan DH, Shankaran V, Dighe AS, et al: Demonstration of an interferon gamma-dependent tumor surveillance system in immunocompetent mice. Proc Natl Acad Sci USA 95: 7556-7561, 1998.

18. Wang S and Koromilas AE: Stat1 is an inhibitor of Ras-MAPK signaling and Rho small GTPase expression with implications in the transcriptional signature of Ras transformed cells. Cell Cycle 8: 2070-2079, 2009.

19. Wang S, Raven JF, Durbin JE and Koromilas AE: Stat1 phosphorylation determines Ras oncogenicity by regulating p27 kip1. PLoS One 3: e3476, 2008.

20. Khodarev NN, Roach P, Pitroda SP, et al: STAT1 pathway mediates amplification of metastatic potential and resistance to therapy. PLoS One 4: e5821, 2009.

21. Rickardson L, Fryknas M, Dhar S, et al: Identification of molecular mechanisms for cellular drug resistance by combining drug activity and gene expression profiles. Br J Cancer 93: 483-492, 2005

22. Pitroda SP, Wakim BT, Sood RF, et al: STAT1-dependent expression of energy metabolic pathways links tumour growth and radioresistance to the Warburg effect. BMC Med 7: 68, 2009.

23. Chun Y, Park B, Koh W, et al: New centromeric component CENP-W is an RNA-associated nuclear matrix protein that interacts with nucleophosmin/B23 protein. J Biol Chem 286: 42758-42769, 2011.

24. Okuwaki M: The structure and functions of NPM1/Nucleophsmin/B23, a multifunctional nucleolar acidic protein. J Biochem 143: 441-448, 2008.

25. Grisendi S, Mecucci C, Falini B and Pandolfi PP: Nucleophosmin and cancer. Nat Rev Cancer 6: 493-505, 2006.

26. Khodarev NN, Roizman B and Weichselbaum RR: Molecular pathways: interferon/stat1 pathway: role in the tumor resistance to genotoxic stress and aggressive growth. Clin Cancer Res 18: 3015-3021, 2012.

27. Velasco-Velazquez M, Jiao X, De La Fuente M, et al: CCR5 antagonist blocks metastasis of basal breast cancer cells. Cancer Res 72: 3839-3850, 2012.

28. Kitamura T, Fujishita T, Loetscher $\mathrm{P}$, et al: Inactivation of chemokine (C-C motif) receptor 1 (CCR1) suppresses colon cancer liver metastasis by blocking accumulation of immature myeloid cells in a mouse model. Proc Natl Acad Sci USA 107: 13063-13068, 2010.

29. Lee JH, Kim HN, Kim KO, et al: CXCL10 promotes osteolytic bone metastasis by enhancing cancer outgrowth and osteoclastogenesis. Cancer Res 72: 3175-3186, 2012.

30. Nakano M, Fujii T, Hashimoto M, et al: Type I interferon induces CX3CL1 (fractalkine) and CCL5 (RANTES) production in human pulmonary vascular endothelial cells. Clin Exp Immunol 170: 94-100, 2012.

31. Nardi V, Naveiras O, Azam M and Daley GQ: ICSBP-mediated immune protection against BCR-ABL-induced leukemia requires the CCL6 and CCL9 chemokines. Blood 113: 3813$3820,2009$.

32. Ohmori Y and Hamilton TA: Cooperative interaction between interferon (IFN) stimulus response element and kappaB sequence motifs controls IFN gamma- and lipopolysaccharide-stimulated transcription from the murine IP-10 promoter. J Biol Chem 268: 6677-6688, 1993. 